\title{
Diagnostic Peritoneal Lavage - A Lost Art? A Retrospective Observational Study at a Canadian Tertiary Trauma Centre
}

\author{
Melanie L Di Quinzio ${ }^{1}$, David A Petrie², John M Tallon ${ }^{3}$ \\ ${ }^{1}$ Department of Medicine, Ottawa General Hospital \\ ${ }^{2}$ Department of Emergency Medicine, Dalhousie University \\ ${ }^{3}$ Department of Emergency Medicine \& Surgery, Nova Scotia Trauma Program
}

\begin{abstract}
INTRODUCTION: Abdominal trauma is a leading cause of morbidity and mortality in the young, adult patient in North America. The standard tools to assess the severity of abdominal trauma have been evolving. The continuing role of diagnostic peritoneal lavage (DPL) as a clinical tool to evaluate intra-abdominal injury today remains controversial. This study investigated trends in the use of DPL, CT (computerized tomography) and FAST (Focused Assessment with Sonography for Trauma) in trauma patients in the province of Nova Scotia's single adult tertiary trauma centre.
\end{abstract}

METHODS: We conducted a retrospective analysis of all patients over the age of 15 with severe blunt and/or penetrating abdominal trauma from 1996 to 2001 who presented to a tertiary care hospital in Nova Scotia. Patients included in this study were those with an injury severity score (ISS) of $\geq 9$ for penetrating trauma and $\geq 12$ for blunt trauma and were derived from the Nova Scotia Trauma Registry. Descriptive statistics were utilized to determine trends over the six year period.

RESULTS: Of 172 patients included in the final cohort, the majority were male and under the age of 50 . Blunt abdominal injury was far more common than penetrating abdominal injury in this cohort. Abdominal CT, FAST, and DPL were performed in $43.6 \%, 19.8 \%$ and $0.6 \%$ of the patients respectively. Absolute numbers of CTs and ultrasounds performed for abdominal trauma increased; however, the percentage of patients receiving those investigations remained constant.

DISCUSSION: Despite having excellent published test characteristics and extensive literature supporting its use, DPL is not commonly used today to assess blunt/penetrating abdominal injury in major trauma patients in Nova Scotia's tertiary trauma centre. These trends may have implications for guideline formulation for care of the major trauma patient with suspected intra-abdominal injuries.

\section{Introduction}

In the United States, injuries are the third leading cause of death, accounting for $6 \%$ of all deaths. ${ }^{1,2}$ Injuries, excluding adverse events in medical care, are the leading cause of death for Canadians between the ages of 1 and 44 and the fourth leading cause of death for Canadians of all ages. ${ }^{3}$ Trauma is common and its treatment often demands timely diagnostic evaluation by health care practitioners. Prompt detection of intra-abdominal hemorrhage is critical in optimizing treatment and improving outcomes for injured patients, and yet accurate diagnosis of intra-abdominal injury remains one of the most challenging aspects of evaluating patients who have sustained blunt or even penetrating trauma ${ }^{4}$ Hemodynamic instability with signs of diffuse peritoneal irritation is an absolute indication for an emergent laparotomy. For those patients that are hemodynamically stable or unstable with other injuries such as major pelvic fractures, (and particularly in those with a compromised sensorium due to injury or medications), the optimal process of diagnostic evaluation is not as immediately clear. Physical examination, diagnostic peritoneal lavage (DPL), computed tomography (CT) and/or Focused Assessment with Sonography for Trauma (FAST) ${ }^{5}$ are used to evaluate blunt and penetrating abdominal trauma patients. Each test has its own particular strengths, weaknesses and test characteristics for evaluating abdominal trauma and these may vary depending upon the clinical scenario and underlying pathology as well as operator-dependant issues. Despite the clear ascendancy of FAST as the bedside test of choice for evaluation of hemoperitoneum, DPL remains well studied and with excellent test characteristics. DPL is still taught as part of the Advanced Trauma Life Support course of the American College of Surgeons in both lecture and practicum format. ${ }^{6}$ Thus, the diagnostic evaluation of these challenging patients remains controversial. ${ }^{7}$

In trauma care, DPL is a safe and effective technique for diagnosing intra-abdominal injury in cases of blunt and penetrating abdominal trauma. ${ }^{7,8}$ DPL is a very rapid and sensitive test with sensitivity of $96-100 \%$ in many series. ${ }^{9-14}$ Nagy et al. reported a sensitivity, specificity and accuracy of $94.4 \%, 99 \%$ and $98.1 \%$ respectively. ${ }^{13}$ The clinical relevance and utility of this diagnostic tool has been well demonstrated and firmly incorporated into trauma practise. Since its introduction, DPL has decreased 
the number of trauma deaths in severely injured patients. ${ }^{15}$ The accepted criteria for a positive DPL gives the test high sensitivity but with specificity that can result in an increase in nontherapeutic laparotomies. Because of this increase in nontherapeutic laparotomies and because DPL is a relatively invasive procedure, its use as the primary bedside test for hemoperitoneum and intra-abdominal injury has declined over recent years with the advent of the non-invasive bedside FAST exam. ${ }^{11}$

In North America, CT of the abdomen is currently the most commonly used diagnostic test for the hemodynamically stable patient in need of evaluation after blunt abdominal trauma. ${ }^{11}$ Abdominal CT effectively identifies free blood and solid organ injuries; however, it is less effective in diagnosing diaphragmatic, pancreatic, and hollow viscus injuries. ${ }^{14,16}$ Despite its many advantages, abdominal CT is a relatively costly and time-consuming diagnostic tool that is contraindicated in unstable and critically ill patients. ${ }^{11}$

More recently, beginning in late 1980s and through the 1990s, trauma sonography or FAST has been used to screen blunt abdominal trauma patients for hemoperitoneum. This method of investigation allows rapid evaluation of more than one torso cavity with sonography (and a cardiac window for evaluation of the presence of pericardial blood). FAST can accurately identify hemoperitoneum; however, it is not as accurate as CT in identifying solid organ injuries. ${ }^{16,17}$ Although sonography has the advantage of being non-invasive, its superior or equivalent sensitivity in comparison to DPL has not been consistently demonstrated. ${ }^{11}$ Several studies have reported higher missed injury rates in FAST exams versus DPL. ${ }^{16}$

Rapidly identifying hemoperitoneum among stable and unstable blunt trauma victims is crucial for early intervention and definitive operative management. Emergency physicians and surgeons originally used DPL in this role, but practices have changed over time. ${ }^{7}$ The current role of DPL as a clinical diagnostic tool to evaluate intra-abdominal injury remains uncertain and evolving. ${ }^{18}$ The purpose of this study was to investigate trends in the use of DPL, CT scan and FAST examination to evaluate patients with blunt and penetrating abdominal trauma in a tertiary care trauma centre with the hypothesis that DPL has become a seldom used tool and that FAST has superseded DPL.

\section{Methods}

Patients were identified for inclusion in this study by performing a prespecified search of the Nova Scotia Trauma Registry database. All patients treated in the sole adult tertiary care centre in Nova Scotia between January 1996 and December 2001 who were older than 15, alive upon arrival to the emergency department and had an injury severity score
(ISS) $\geq 9$ for penetrating trauma and $\geq 12$ for blunt trauma overall and had abdominal injury were included in the study as per the retrospective criteria of inclusion within the Nova Scotia Trauma Registry. This cohort included those patients who presented primarily to the tertiary trauma centre as well as those who were transferred from other hospitals by ground or air transport. DPL, CT scan and FAST examinations were included in this study when performed at the tertiary trauma centre (i.e., imaging performed at a sending institution were not included). Abdominal injuries were identified through the trauma registry as all injuries to the abdomen and/or pelvic contents excluding superficial skin, subcutaneous or muscle injuries (AIS codes 515099 - 545626). The patients may have had other serious trauma injuries (i.e., extremity injuries, and/or CNS injuries) but these were not analyzed in this study. This study period was chosen since it corresponded to institution of a new provincial trauma system ranging from a comprehensive re-organization of the EMS system to formal organization of a dedicated trauma team and trauma team leaders for the tertiary trauma centre as described elsewhere. ${ }^{19}$ However, during the study period there was no effort nor policy to formalize the diagnostic evaluation of the study cohort of patients and all three modalities of evaluation (DPL, FAST and CT) were available to trauma team leaders and the trauma team. The province's sole adult tertiary trauma centre has a full provincial and regional trauma care mandate and dedicated 24/7 trauma team availability with dedicated trauma team leaders as described elsewhere. ${ }^{20}$

This was a retrospective observational study using data from the Nova Scotia Trauma Registry. Inclusion criteria for the trauma registry ${ }^{21}$ are presented in Appendix A. All injured patients presenting to the tertiary care centre are screened for potential inclusion in the trauma registry. The data collected in the Trauma Registry Database include facts related to the nature of the injury and trauma event, patient demographics, patient care, and patient outcomes. ${ }^{21,22}$ Trauma registry data is collected by a team of healthcare professionals (nurses, paramedics, health records personnel) with training in the Abbreviated Injury Score (AIS), the trauma registry software (Collector ${ }^{\mathrm{TM}}$ ), the World Health Organization International Classification of Diseases (ICD-10) and Canadian Classification of Health Interventions (CCI). The registry software provides automated internal edit checks to ensure that dates and times are consistent as well as to ensure that no invalid codes are entered. Data consistency is also examined visually by the provincial trauma registry coordinator. A thorough reabstracting audit is regularly conducted of $10 \%$ of randomly-selected cases. Further, an error report is generated by the National Trauma Registry based on the data submitted from the Nova Scotia Trauma Registry to identify coding errors not identified through provincial screening protocols. Nova Scotia has a provincewide population based trauma system which includes one 
adult ( $\geq 16$ years) tertiary trauma centre (the study site) similar to a level I American trauma centre in many ways and the registry has been successfully used in other peer reviewed published research. . $^{18,20,23}$

This project employed secondary analysis of an existing database, and involved no patient contact, treatment or chart reviews. Simple descriptive statistics were utilized to describe trends in the use of DPL, CT and FAST as diagnostics tools to evaluate these patients. Analysis of the data was conducted by calculating incidences for each of the diagnostic procedures. The statistical software utilized to perform the analysis was SAS version 8. Ethics approval was granted by the Capital Health Research Ethics Board, the research ethics board for the trauma centre.

\section{Results}

A total of 172 patients were identified using the Nova Scotia Trauma Registry for the study period (Table 1). Of these 172 identified patients, $2 / 3$ were male and the largest proportion of patients was between the ages of 20 and 29 $(22.7 \%)$. Most of the abdominal injuries were blunt in nature $(90.7 \%)$ and the majority of these occurred in Nova Scotia residents between the years 1999 and 2001. The majority of the patients had an ISS between 12 and 30 with $16.3 \%$ having an ISS of 40 or over.

The total amount of diagnostic procedures per patient was relatively low. Of the patients presenting to the tertiary trauma centre between January 1996 and December 2001, 43.6\%, $19.8 \%$ and $0.6 \%$ received a CT scan, FAST examination or DPL respectively (Table 2). Most of these patients received only one method of imaging of their abdomen with 13 of the $172(7.6 \%)$ patients having two methods and none with all three. Nearly half of the patients had no imaging of their abdomen (45.3\%). Of the patients who underwent FAST for investigation of abdominal trauma -12 of the 34 also underwent CT (35.3\%). Most patients who underwent a CT abdomen had no other investigations (84\%). The only patient who underwent DPL also underwent FAST during this study and this was a patient with hemodynamic instability in the setting of pelvic trauma. Patients in the study cohort also received other imaging modalities for concurrent injuries such as CT head (55.2\%), CT thorax (26.2\%) and CT pelvis $(29.7 \%)$. Of the 78 patients without abdominal imaging, $1 / 3$ underwent some form of urgent surgical exploration/ intervention. Just under $1 / 2$ of the patients with abdominal imaging also had urgent surgical interventions (Table 3 ).

The absolute number of CT scans performed has increased over the last years of the study (two in 1996 versus 32 in 2001); however, the percentage of patients presenting with blunt or penetrating abdominal trauma receiving CT scans
Table 1. Patient demographics.

\begin{tabular}{llll}
\hline Total Patients & $172(\%)$ & $\begin{array}{c}\text { Province } \\
\text { Nova Scotia } \\
\text { Other }\end{array}$ & $157(91.3)$ \\
$\begin{array}{l}\text { Gender } \\
\text { Females }\end{array}$ & $58(33.7)$ & $\begin{array}{c}\text { Year } \\
15(8.7)\end{array}$ \\
$\quad$ Males & $114(66.3)$ & 1996 & $7(3.8)$ \\
Age & & 1997 & $7(3.8)$ \\
$<20$ & $28(16.3)$ & 1998 & $6(3.3)$ \\
$20-29$ & $39(22.7)$ & 1999 & $20(11.0)$ \\
$30-39$ & $34(19.8)$ & 2000 & $62(34.1)$ \\
$40-49$ & $26(15.1)$ & 2001 & $70(38.5)$ \\
$50-59$ & $17(9.9)$ & Index Severity Score & $7(4.1)$ \\
$\geq 60$ & $28(16.3)$ & $<12$ & $56(33.7)$ \\
Injury Type & & $12-20$ & $58(33.7)$ \\
Blunt & $156(90.7)$ & $20-30$ & $23(13.4)$ \\
Penetrating & $16(9.3)$ & $30-40$ & $28(16.3)$ \\
\hline
\end{tabular}

Table 2. Modes of imaging.

\begin{tabular}{ll}
\hline Total Patients & $172(\%)$ \\
& \\
No Imaging & $78(45.3)$ \\
CT Abdomen alone & $63(36.6)$ \\
CT and FAST & $12(7.0)$ \\
CT and DPL & $0(0.0)$ \\
FAST alone & $21(12.2)$ \\
FAST and DPL & $1(0.6)$ \\
CT Head & $95(55.2)$ \\
CT Thorax & $46(26.2)$ \\
CT Pelvis & $51(29.7)$ \\
\hline
\end{tabular}

Table 3. Surgical intervention rates.

\begin{tabular}{lccc}
\hline & $\begin{array}{c}\text { No Surgical } \\
\text { Intervention }\end{array}$ & $\begin{array}{c}\text { Surgical } \\
\text { Intervention }\end{array}$ & Total \\
\hline $\begin{array}{l}\text { No Abdominal } \\
\text { Imaging }\end{array}$ & $52(66.7 \%)$ & $26(33.3 \%)$ & 78 \\
$\begin{array}{l}\text { Abdominal } \\
\text { Imaging }\end{array}$ & $54(57.4 \%)$ & $40(42.6 \%)$ & 94 \\
\hline
\end{tabular}

(approximately $40 \%$ per year) has not (Figures 1 and 2). The average annual percentage of patients undergoing CT scans during this study was $40.8 \%$ with a standard deviation of $9.6 \%$. There was no significant difference between the proportion of CT scans done in any of the years from 1998 to 2001. There were a total of 34 FAST examinations performed during the study period, which made it a far more common clinical test than DPL (one performed) but still less than half as common as the use of CT scans. The absolute number of FAST examinations increased over the last three years of the study, but the annual proportion of FAST examinations was not significantly different for the years 1999 to 2001 .

\section{Discussion}

Prompt detection of intra-abdominal hemorrhage is critical in optimizing treatment and improving outcomes for injured patients, and yet accurate diagnosis of this injury pattern remains one of the most challenging aspects of evaluating patients who have sustained blunt or penetrating trauma. Emergency physicians, trauma team leaders and 


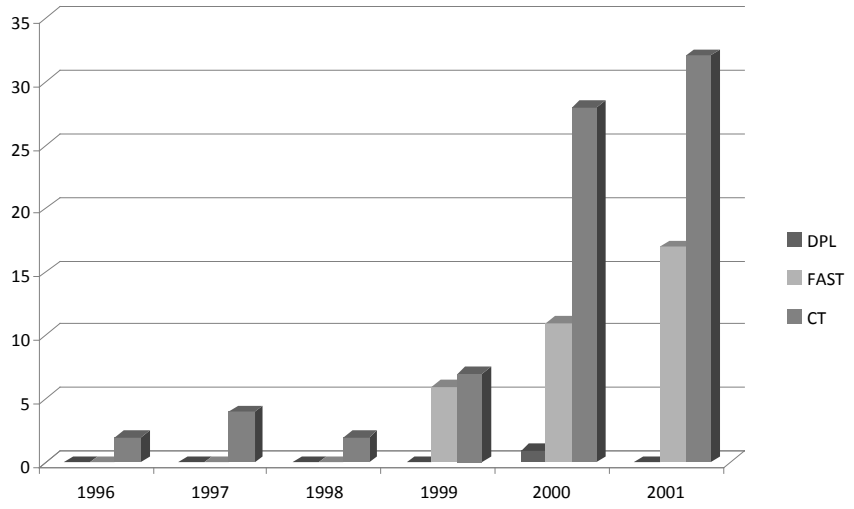

Figure 1. Absolute number of investigations performed per year.

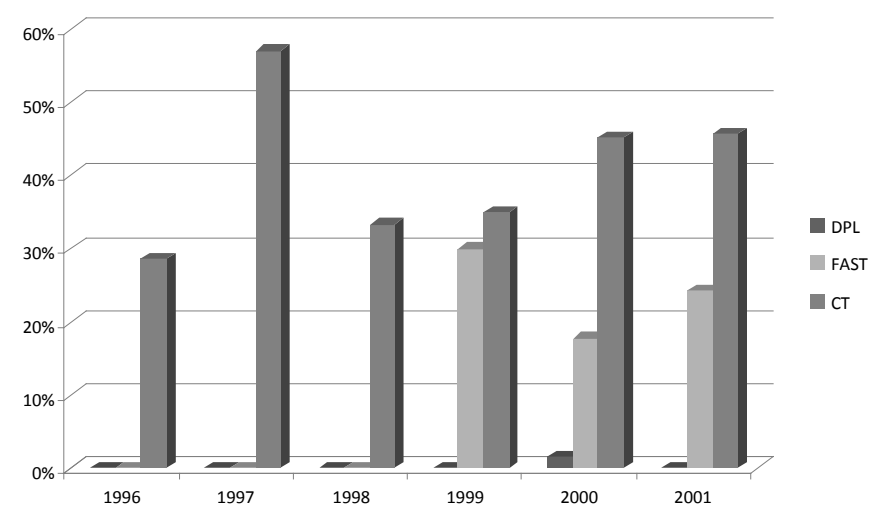

Figure 2. Percentage of patients receiving each investigation per year.

trauma surgeons make every effort to diagnose these injuries in a timely fashion and often rely on DPL, CT scan or FAST examination to aid in their evaluation. With the development of bedside ultrasound skills on the part of clinicians and the evolution of advanced and rapid CT scanning, controversy exists regarding any residual role of DPL in the assessment of the trauma patient with suspected significant abdominal injury. Despite this, DPL diagnostic evaluation is still taught as an integral part of the ATLS course by the American College of Surgeons. The purpose of our paper was simply to demonstrate the current low prevalence of DPL use and suggest that the role of DPL has declined in a provincial tertiary trauma centre in conjunction with the far more prevalent use of FAST and CT. While assumed by most trauma practitioners, this has not been clearly shown in previous studies. The frequency of DPL prior to January of 1996 could not be assessed from our database, so comments on the change of practice prior to that time are suppositional, although it is appreciated that DPL was the only immediately available test for hemoperitoneum at the bedside during the resuscitative phase of care at that time. Whether or not DPL does have a residual, albeit small and infrequent role in the evaluation of abdominal trauma in the modern trauma centre is beyond the scope of this paper, although it has been suggested that it may still be of use. A recent paper by Friese et al, suggests that DPL may play a key role in the evaluation of the patient with pelvic fracture and hemodynamic instability due to the presence of a significant false negative rate for FAST in their retrospective review. ${ }^{24}$ However, an invasive diagnostic test (DPL), which is seldom performed, brings to attention questions of skills maintenance and diagnostic utility of seldom used laboratory indices. ${ }^{19}$ In our study period, despite being a teaching centre and a tertiary trauma centre with a significant blunt pelvic fracture population, we performed only one DPL - hardly the clinical exposure needed for both clinicians and trainees to maintain skills in performing this test or interpreting its results.

In our trauma centre, the use of CT scanning has become the dominant form of investigation for patients presenting to the emergency department with blunt or penetrating abdominal injury and without obvious immediate need for operative intervention. Over the last few years of this study the absolute number of CT scans increased, although the percentage of patients receiving this investigation did not significantly change. This trend indicates that there has not been a shift in the protocol to evaluate these patients, but rather an increase in the total number of major trauma patients presenting to the emergency department as confirmed by the trauma program's annual reports (total of 100 major trauma cases in 1994-1995 versus 400 cases in 2004-2005). ${ }^{21}$ More recently, it has been observed that up to $90 \%$ of trauma team activations (in 2006-2007) utilized full CT (head to pelvis) evaluation of the trauma patient ( $90 \%$ if directly brought to trauma centre by EMS and $80 \%$ if brought via another intermediate regional trauma centre) (personal communication, Ms. Beth Sealy, NS Trauma Registrar, October 2007). In this study, the total number of FAST examination increased between 1999 and 2002; however, the percentage of patients receiving this investigation did not. This trend again indicates that a significant change in diagnostic practice is not occurring and that an actual increase in FAST examinations reflects an increase in the major trauma patient population arriving for evaluation at our tertiary care centre. The use of diagnostic laparoscopy for evaluation of intra-abdominal injury was not utilized at our tertiary care centre during the study period but has been well described in the trauma population and especially in the assessment of penetrating injuries to the abdomen. ${ }^{25}$ The increased use of this diagnostic tool may also contribute to the demise of DPL in other tertiary trauma centres that see a large cohort of penetrating trauma cases. 


\section{Limitations}

This study of imaging trends in abdominal trauma has several limitations. It is a retrospective analysis using a trauma database and is subject to all the limitations of retrospective reviews and studies predicated on registry data. It is a relatively small study with a large variation in traumas that met the inclusion criteria from year to year (range seven cases per annum to 70 per annum). We feel that this variation in case load reflects the trauma system implementation and maturity that occurred in this province, which prior to 1996-1997 lacked any formal trauma system; the trauma system implementation was comprehensive, province-wide and has been shown to have significantly increased the number of major trauma patients being brought to tertiary care in the post implementation period. ${ }^{18}$ Another limitation of this study is the effect of concurrent injuries as all patients with abdominal injury were included regardless of their other, potentially significant, injuries. This was intentional, as the design of the study was to capture how all abdominal traumas were investigated in the emergency/ resuscitation period of patient evaluation.

This study was also limited by the authors' inability to determine the rationale or decision making process inherent with patient evaluation. In the cases where more than one imaging modality was employed to investigate the abdomen injury, assumptions as to the reason to pursue a second imaging technique cannot be made by the authors. Where imaging of the abdomen was not performed at all, one could assume the patient had more severe injuries that took precedence over imaging the abdomen. The purpose of this paper was not to devise or evaluate a protocol for imaging of the injured abdomen but rather to simply document an assumed, but unproven, decline in DPL utilization at our provincial tertiary trauma centre.

\section{Summary}

In summary, CT scanning is the dominant form of diagnostic investigation used in this Canadian trauma centre for adult patients (older than 15 years) presenting to the emergency department for blunt or penetrating abdominal trauma assessment. This result is consistent with other such centres in North America. In addition, our data demonstrate that the overall use of the FAST exam began to increase in the early 2000s in this centre and the use of DPL appears to be a "dying art" and is seldom, if ever, employed to assess abdominal trauma in this centre. To the best of the authors' knowledge, this is the first paper to document a clear decline (and extinction?) of DPL as a bedside diagnostic tool in the major trauma patient in a Canadian tertiary trauma centre. Current practise guidelines and ATLS ${ }^{\text {TM }}$ teaching still include the use of DPL which may not reflect current practise. A role for DPL in the unstable patient with major pelvic injury and a negative FAST may still exist but our data indicate that skills maintenance and interpretive issues of the DPL result may be a significant issue, even in a teaching centre/tertiary trauma centre.

\section{Appendix A. Inclusion criteria of the Nova Scotia Trauma Program Registry Major Injury Dataset}

Inclusion in the dataset is based on the following:

Injury resulting from trauma and associated with an injury included in the ICD-10-CA external cause of injury codes and:

- $\quad$ ISS $\geq 12$ for blunt or burn trauma, ISS $\geq 9$ for penetrating trauma, or

- Trauma team activation, or

- Death in the emergency department, or

- Death within 24 hours of admission, or

- Death at the scene, or

- Inclusion based on treatment and admission at another trauma centre followed by transfer to a second or third trauma centre for ongoing care of the initial injury

\section{Acknowledgements}

The authors of this study would like to extend a personal thank you to Ms. Corinne Burke, for her expert preparation of the manuscript.

\section{REFERENCES}

1. Fingerhut L, Warner M. Injury Chartbook: Health United States. 1997. Hyattsville MD, National Center for Health Statistics.

2. Wynn A, Wise M, Wright MJ, Rafaat A, Wang YZ, Steeb G, McSwain $\mathrm{N}$, Beuchter KJ, Hunt JP. Accuracy of administrative and trauma registry databases. J Trauma. 2001;51:464-468.

3. Public Health Agency of Canada. Facts on injury. 2007. 4-11-0007.

4. Miller MT, Pasquale MD, Bromberg WJ, Wasser TE, Cox J. Not so FAST. J Trauma. 2003;54:52-59.

5. Dietrich J, Heller MB. The FAST Exam. In: Jone RA, Welch RD, editors. Ultrasonagraphy in Trauma. Dallas Texas: College of Emergency Physicians, 2004.

6. ATLS, Student Course Manual. 7th ed. Chicago Ill: American College of Surgeons, 2007.

7. American College of Surgeons. Abdominal Trauma. ATLS Student Course Manual. Chicago Ill: American College of Surgeons, 2007.

8. Brakenridge SC, Nagy KK, Joseph KT, An GC, Bokhari F, Barrett J. Detection of intra-abdominal injury using diagnostic peritoneal lavage after shotgun wound to the abdomen. J Trauma. 2003;54:329-331.

9. Blow O, Bassam D, Butler K, Cephas GA, Brady W, Young JS. Speed and efficiency in the resuscitation of blunt trauma patients with multiple injuries: the advantage of diagnostic peritoneal lavage over abdominal computerized tomography. J Trauma. 1998;44:287-290.

10. Day AC, Rankin N, Charlesworth P. Diagnostic peritoneal lavage: integration with clinical information to improve diagnostic performance. J Trauma. 1992;32:52-57.

11. Gonzalez RP, Ickler J, Gachassin P. Complementary roles of diagnostic peritoneal lavage and computed tomography in the evaluation of blunt abdominal trauma. J Trauma. 2001;51:1128-1134.

12. Henneman PL, Marx JA, Moore EE, Cantrill SV, Ammons LA. Diagnostic peritoneal lavage: accuracy in predicting necessary laparotomy following blunt 
and penetrating trauma. J Trauma. 1990;30:1345-1355.

13. Nagy KK, Roberts RR, Joseph KT, Smith RF, An GC, Bokhari F, Barrett J. Experience with over 2500 diagnostic peritoneal lavages. Injury. 2000;31:479482.

14. Schurink GW, Bode PJ, van Luijt PA, van Vugt AB. The value of physical examination in the diagnosis of patients with blunt abdominal trauma: a retrospective study. Injury. 1997;28:261-265.

15. Hughes TM. The diagnosis of gastrointestinal tract injuries resulting from blunt trauma. Aust N Z J Surg. 1999;69:770-777.

16. Liu M, Lee C-H, P'eng F-K. Prospective comparison of diagnostic peritoneal lavage, computed tomographic scanning, and ultrasonography for the diagnosis of blunt abdominal trauma. J Trauma. 1993;35:267-270.

17. Stafford RE, McGonigal MD, Weigelt JA, Johnson TJ. Oral contrast solution and computed tomography for blunt abdominal trauma: a randomized study. Arch Surg. 1999;134:622-626.

18. Tallon JM, Fell DB, Ackroyd-Stolarz S, Petrie D. Influence of a new provincewide trauma system on motor vehicle trauma care and mortality. J Trauma. 2006;60:548-552.

19. Bergeron E, Lavoie A, Belcaid A, Moore L, Clas D, Razek T, Lessard J, Ratte L. Surgical management of blunt thoracic and abdominal injuries in Quebec: a limited volume. J Trauma. 2007;62:1421-1426.

20. Ahmed JM, Tallon JM, Petrie DA. Trauma management outcomes associated with nonsurgeon versus surgeon trauma team leaders. Ann Emerg Med. 2007;50:7-12, 12.

21. EHS Nova Scotia Trauma Program. Internet. 2007. 1-4-0007.

22. Government of Nova Scotia. EHS. Internet. 2007.

23. Tallon JM, Ahmed JM, Sealy B. Airway management in penetrating neck trauma at a Canadian tertiary trauma centre. CJEM. 2007;9:101-104.

24. Friese RS, Malekzadeh S, Shafi S, Gentilello LM, Starr A. Abdominal ultrasound is an unreliable modality for the detection of hemoperitoneum in patients with pelvic fracture. J Trauma. 2007;63:97-102.

25. Weinberg JA, Magnotti LJ, Edwards NM, Claridge JA, Minard G, Fabian TC, Croce MA. "Awake" laparoscopy for the evaluation of equivocal penetrating abdominal wounds. Injury. 2007;38:60-64.

\section{Cape Breton Island:Great People,}

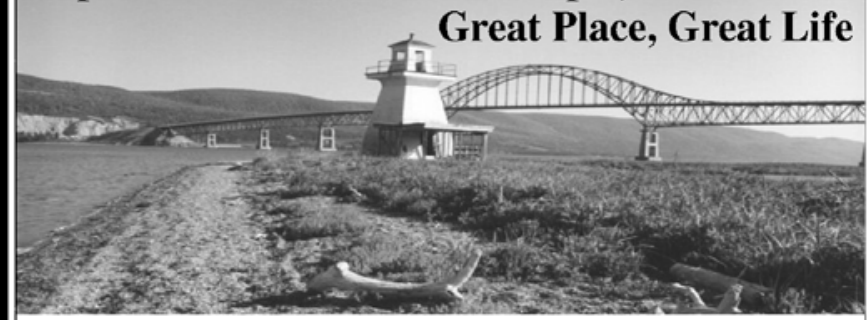

The Cape Breton District Health Authority has openings available in:

-Emergency Medicine

•Child Psychiatry

-Vascular Surgery •General Surgery - Rural Family Medicine (Neil's Harbour)

The District serves more than 130,000 people through regional, community and rural hospitals, Continuing Care, Mental Health Services, Addiction Services, Public Health Services and the Cape Breton Cancer Centre. Cape Breton's unique beauty, diverse culture and year-round recreational opportunities make the Island a vibrant place to work and play.

All candidates must be certified by the Royal College of Physicians and Surgeons and be eligible for licensure in the province of Nova Scotia.

\section{Inquiries and applications to:}

Dr. M.A. Naqvi, Medical Director

Cape Breton District Health Authority

1482 George Street

Sydney, Nova Scotia B1P 1P3

Fax: (902) 567-7255

E-mail: naqvim@cbdha.nshealth.ca

Website: www.cbdha.nshealth.ca
Cape Breton District HEALTH AUTHORITY Making Heaithier Choices Together
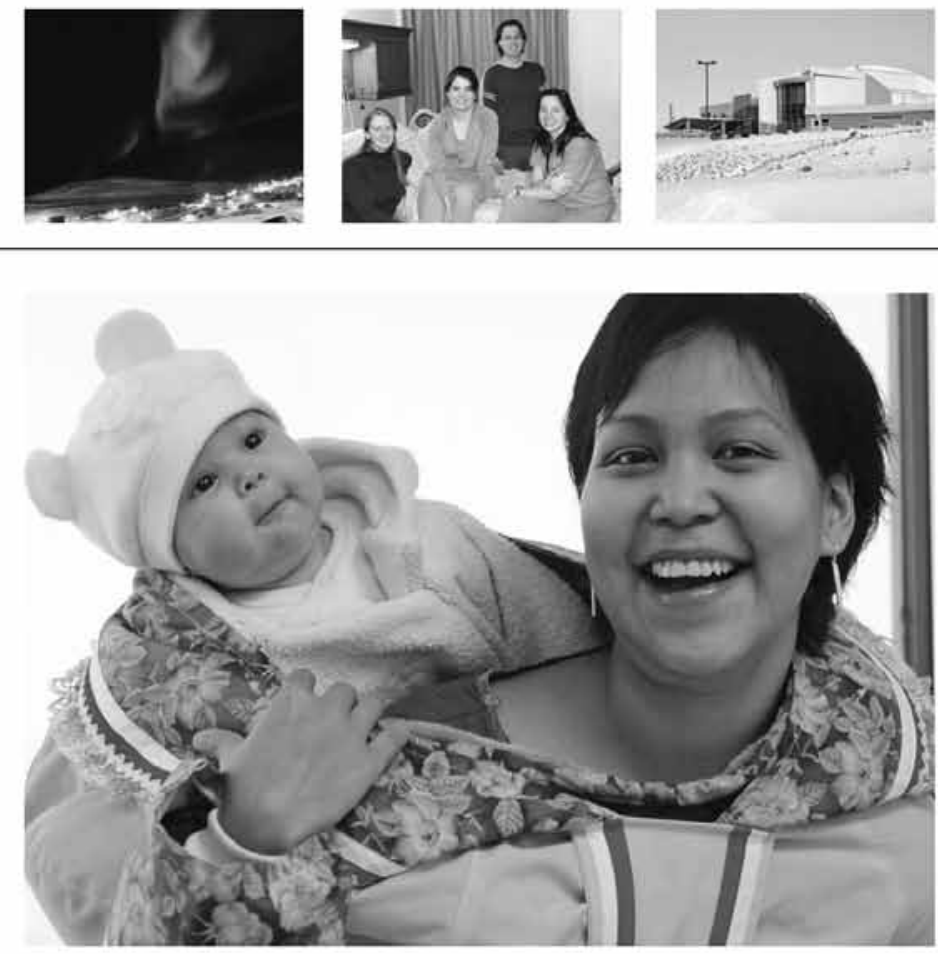

JOIN US IN NUNAVUT FAMILY PRACTITIONERS

This is your opportunity to begin your medical career in Nunavut in a collaborative family practice. Experience the rich Inuit culture, breathtaking scenery, and a change of pace

One of our regular physicians describes the practice like this: "I chose to start my career as a family physician in lqaluit after a rotation here as a resident. I was attracted to the opportunity to practice full service family medicine. I've expanded my clinical skills, learned about Inuit culture and worked with a supportive group of physicians from across the country".

The Government of Nunavut offers competitive rates and travel expenses. Short or long term contract available

For more information, Please contact Physician Services:

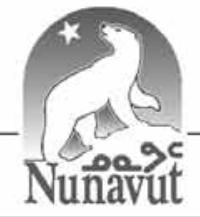

